\title{
Uniqueness of the functional approach
}

\author{
H. O. Girotti and T. J. M. Simões \\ Instituto de Física, Universidade Federal do Rio Grande do Sul, 90000 Porto Alegre, RS, Brazil \\ (Received 8 January 1980; revised manuscript received 22 April 1980)
}

\begin{abstract}
It is shown that the phase-space Schwinger equations can only be appropriately formulated in terms of a specific discrete procedure. Nevertheless, the solutions to Schwinger equations turn out to be independent of the discrete procedure. We stress the point that a multiplicity of representations for the quantum-mechanical amplitudes is not a peculiar effect of the time-slicing definition of the path integral, but rather a necessity of the functional approach.
\end{abstract}

\section{INTRODUCTION}

The fact that the quantum-mechanical propagator corresponding to a physical system fully specified by a Hamiltonian operator $H$ does not possess a unique representation in terms of canonical path integrals has already been recognized. ${ }^{1-11}$ In fact, for defining the phase-space path integral through the time-slicing method ${ }^{12,13}$ one is required to choose a particular discrete procedure. Since there is a continuous infinity of discrete methods at our disposal, ${ }^{2}$ a nonunique path-integral representation for the matrix elements of the quantum theory naturally emerges.

Of course, the path integral must, on evaluation, be independent of the discrete procedure, since it is derived from a fixed quantum-mechanical Hamiltonian. That this is so has been repeatedly checked in the literature ${ }^{10,14,15}$; nevertheless, the time-slicing definition of the phase-space path integral has been considered as ambiguous because it gives rise to a multiplicity of representations for the quantum-mechanical amplitudes. ${ }^{16,17}$

Our main purpose in this work is to show that alternative definitions for the canonical path integral also lead to a nonunique representation for the propagator. This is a consequence of the fact that, as we shall demonstrate, the functional differential scheme of Schwinger ${ }^{18,19}$ can only be appropriately formulated after specifying a discrete method. Since Feynman's canonical path inte$\operatorname{gral}^{12,13}$ can be seen as the functional Fourier transform solving Schwinger's differential equations ${ }^{18,19}$ its structural dependence upon the discrete method follows from general theoretical considerations. What appears to be linked with the particular definition adopted for the path integral is the operational level at which the terms dependent on the discrete procedure cancel out. This is our Sec. II.

The relevant points are discussed throughout the paper and in Sec. III, where some final remarks are made.

\section{DEPENDENCE OF SCHWINGER'S EQUATIONS ON DISCRETE PROCEDURE}

It has been a common belief that a multiplicity of representations for the quantum-mechanical amplitudes only arises in connection with the timeslicing definition of the path integral. ${ }^{16,17}$ This is not true. In this section we show, on general grounds, that a discrete prescription is always required for an appropriate settlement of the functional formulation of quantum dynamics.

As a first step into this goal we shall demonstrate that, in the more general case, Schwinger's functional differential equations must be formulated in terms of a specific discrete procedure.

To proceed towards the derivation of Schwinger's equations we start by considering a physical system, described by the Hamiltonian operator $H$, in the presence of external sources. The sourcessystem interaction in chosen to be

$$
H^{(s)}=-J \cdot Q-K \cdot P,
$$

where $J$ and $K$ are $c$-number external sources for position $(Q)$ and momentum $(P)$, respectively. Sometimes, and for abbreviation purposes, we shall designate these sources, generically, by $s$. The number of degrees of freedom of the system is $N$. Therefore,

$$
J \cdot Q=\sum_{r=1}^{N} J_{r} Q_{r}, \quad K \cdot P=\sum_{r=1}^{N} K_{r} P_{r} .
$$

The equal-time commutation relations are the usual canonical ones, i.e.,

$$
\begin{aligned}
& {\left[Q_{r}(t), Q_{k}(t)\right]=\left[P_{r}(t), P_{k}(t)\right]=0,} \\
& {\left[Q_{r}(t), P_{k}(t)\right]=i \hbar \delta_{r k}, \quad r, k=1, \ldots, N .}
\end{aligned}
$$

From now on we set $\hbar=1$.

We shall be working in a picture where the time evolution of observables $(L)$ is governed by $H$ while the sources-system interaction is carried out by the state vector $(|\psi\rangle)$. This is the fictitious sources picture whose defining equations are $\mathrm{e}^{18,19}$ 


$$
\begin{aligned}
& \frac{d L}{d t}=i[H, L], \\
& |\psi(t)\rangle=U\left[s \mid t, t_{i}\right]\left|\psi\left(t_{i}\right)\right\rangle,
\end{aligned}
$$

and the unitary operator $U\left[s \mid t, t_{i}\right]$ is to be determined from

$$
U\left[s \mid t, t_{i}\right]=I-i \int_{t_{i}}^{t} d t^{\prime} H^{(s)}\left(t^{\prime}\right) U\left[s \mid t^{\prime}, t_{i}\right] .
$$

In the limit of vanishing external sources $(s=0)$ the fictitious sources picture coincides with the
Heisenberg picture. From (2.1) and (2.5) one finds that

$$
\begin{aligned}
(i)^{-(l+m)} \frac{\delta^{l+m} U\left[s \mid t_{f}, t_{i}\right]}{\delta J_{k_{1}}\left(t_{1}\right) \cdots \delta J_{k_{l}}\left(t_{l}\right) \delta K_{r_{1}}\left(t_{1}^{\prime}\right) \cdots \delta K_{r_{m}}\left(t_{m}^{\prime}\right)} \\
\quad=\left(t_{f}, T\left\{Q_{k_{1}}\left(t_{1}\right) \cdots Q_{k_{l}}\left(t_{l}\right) P_{r_{1}}\left(t_{1}^{\prime}\right) \cdots P_{r_{m}}\left(t_{m}^{\prime}\right)\right\}, t_{i}\right),
\end{aligned}
$$

where the chronologically ordered $T$ product is defined as follows:

$$
\left(t_{f}, T\left\{Q_{k}(t) P_{r}\left(t^{\prime}\right)\right\}, t_{i}\right)=\left\{\begin{array}{l}
U\left[s \mid t_{f}, t\right] Q_{k}(t) U\left[s \mid t, t^{\prime}\right] P_{r}\left(t^{\prime}\right) U\left[s \mid t^{\prime}, t_{i}\right], \text { if } t>t^{\prime} \\
U\left[s \mid t_{f}, t^{\prime}\right] P_{r}\left(t^{\prime}\right) U\left[s \mid t^{\prime}, t\right] Q_{k}(t) U\left[s \mid t, t_{i}\right], \text { if } t^{\prime}>t .
\end{array}\right.
$$

All subscripts in (2.6) run from 1 to $N$.

Schwinger's equations are the equations of motion for the functional derivatives of $U$ with respect to the external sources. One can verify that

$$
\begin{aligned}
\frac{d}{d t} \frac{1}{i} \frac{\delta U\left[s \mid t_{f}, t_{i}\right]}{\delta J_{r}(t)}= & U\left[s \mid t_{f}, t\right] A_{r}(Q(t), P(t)) U\left[s \mid t, t_{i}\right] \\
& -K_{r}(t) U\left[s \mid t_{f}, t_{i}\right]
\end{aligned}
$$

$$
\begin{aligned}
\frac{d}{d t} \frac{1}{i} \frac{\delta U\left[s \mid t_{f}, t_{i}\right]}{\delta K_{r}(t)}= & U\left[s \mid t_{f}, t\right] B_{r}(Q(t), P(t)) U\left[s \mid t, t_{i}\right] \\
& +J_{r}(t) U\left[s \mid t_{f}, t_{i}\right]
\end{aligned}
$$

where

$$
\begin{aligned}
& A_{r}(Q(t), P(t))=\frac{\partial H(Q(t), P(t))}{\partial P_{r}(t)}, \\
& B_{r}(Q(t), P(t))=-\frac{\partial H(Q(t), P(t))}{\partial Q_{r}(t)} .
\end{aligned}
$$

For arriving to (2.8) we have explicitly used the equal-time canonical commutation relations (2.2). Moreover, the ordering of noncommuting factors is to be maintained during the differentiation process indicated in (2.9).

To complete the derivation of Schwinger's equa tions we must recast the right-hand side of (2.8) in terms of functional derivatives of $U$. Here is, precisely, where the trouble begins. We shall next analyze the situation in detail.

If $A_{r}$ and $B_{r}$ are of the form

$$
F(Q(t))+G(P(t))
$$

where $F$ and $G$ are arbitrary functions of $Q(t)$ and $P(t)$, respectively, one can always write

$$
\begin{aligned}
U\left[s \mid t_{f}, t\right] \times\left\{\begin{array}{l}
A_{r}(Q(t), P(t)) \\
B_{r}(Q(t), P(t))
\end{array}\right\} \times U\left[s \mid t, t_{i}\right] \\
=\left\{\begin{array}{l}
A_{r}\left(\frac{1}{i} \frac{\delta}{\delta J(t)}, \frac{1}{i} \frac{\delta}{\delta K(t)}\right) \\
B_{r}\left(\frac{1}{i} \frac{\delta}{\delta J(t)}, \frac{1}{i} \frac{\delta}{\delta K(t)}\right)
\end{array}\right\} \times U\left[s \mid t_{f}, t_{i}\right] .
\end{aligned}
$$

This is, of course, a consequence of (2.2). However, if $A_{r}$ and/or $B_{r}$ contain products of noncommuting operators one can no longer use (2.11). As we shall see next, the problem can only be solved by choosing a discrete procedure. By this we mean that the operators $A_{r}$ and $B_{r}$ are rewritten as follows:

$$
\begin{aligned}
& A_{r}(Q(t), P(t))=\left[A_{r}(Q(t), P(t))\right]_{\alpha_{r}-\alpha}, \\
& B_{r}(Q(t), P(t))=\left[B_{r}(Q(t), P(t))\right]_{\alpha,-\alpha} .
\end{aligned}
$$

The right-hand sides of Eqs. (2.12a) and (2.12b) are defined by the sequence of mapping $s^{8}$

$$
L(Q, P) \stackrel{\alpha}{\rightarrow} l_{\alpha}(q, p) \stackrel{-\alpha}{\longrightarrow} L_{\alpha,-\alpha}(Q, P),
$$

where

$$
\begin{aligned}
l_{\alpha}(q, p)=\int_{-\infty}^{+\infty} & d v \exp (i p \cdot v) \\
& \times\left\langle q-\left(\frac{1}{2}-\alpha\right) v|L(Q, P)| q+\left(\frac{1}{2}+\alpha\right) v\right\rangle,
\end{aligned}
$$$$
L_{\alpha}(Q, P)=(2 \pi)^{-N} \int_{-\infty}^{+\infty} d q d p l(q, p) \Delta(q, p ; \alpha),
$$

and

$$
\begin{aligned}
\Delta(q, p ; \alpha)=(2 \pi)^{-N} \int_{-\infty}^{+\infty} & d u d v \exp (i \alpha u \cdot v) \\
& \times \exp \{i[(q-Q) \cdot u+(p-P) \cdot v]\} .
\end{aligned}
$$

Here, $L(Q, P)$ and $l(q, p)$ are arbitrary functions. 
We denote operators by capital letters and $c$-number functions by lower case letters. All integrals in Eqs. (2.14)-(2.16) must be understood in $R_{N}$. The parameter $\alpha$ is real and such that

$$
-\frac{1}{2} \leqslant \alpha \leqslant+\frac{1}{2} \text {. }
$$

We shall refer to the set of mappings defined in Eqs. (2.14) and (2.15) as to the generalized Weyl transform with index $\alpha\left(\mathrm{GWT}_{\alpha}\right)$. One can easily verify that the correspondence rules (2.14) and (2.15) follow from those of Agarwal and Wolf ${ }^{2}$ by establishing in Cohen's ${ }^{2-4}$ general ordering scheme

$$
f(u, v)=\exp (i \alpha u \cdot v) .
$$

The properties of the $\mathrm{GWT}_{\alpha}$ were studied in Ref.

8. Here, we only quote that

$$
L_{\alpha,-\alpha}(Q, P)=L_{0,0}(Q, P)=L(Q, P) .
$$

It is through this property [see Eqs. (2.12)] that the discretization prescription comes into the game.

The right-hand sides of Eqs. (2.12) can now be explicitly evaluated, and one obtains

$$
\left\{\begin{array}{c}
A_{r}(Q(t), P(t)) \\
B_{r}(Q(t), P(t))
\end{array}\right\}=(2 \pi)^{-2 N} \int_{-\infty}^{+\infty} d x d y d u d v \times\left\{\begin{array}{c}
a_{r_{\alpha}}(x, y) \\
b_{r_{\alpha}}(x, y)
\end{array}\right\} \times \exp \left[i\left(\frac{1}{2}-\alpha\right) u \cdot v\right] \exp \{i[x-Q(t)] \cdot u\} \exp \{i[y-P(t)] \cdot v\},
$$

where, $a_{r_{\alpha}}$ and $b_{r_{\alpha}}$ are the GWT $\mathrm{GW}_{\alpha}$ of $A_{r}$ and $B_{r}$, respectively. Therefore, the GWT $\mathrm{G}_{\alpha}$ reduces any arbitrarily complicated ordering problem to that of a product of a function of $Q$ times a function of $P$. This is the crucial role played by the discrete procedure. Since

$$
F(Q(t)) G(P(t))=\lim _{t^{\prime} \rightarrow t} T\left\{F(Q(t)) G\left(P\left(t^{\prime}\right)\right)\right\},
$$

where $t_{-}=t-\delta, \delta>0$, the replacement of (2.20) into (2.8) lead us, in view of (2.6), into the following final form for Schwinger's equations:

$$
\begin{aligned}
& \frac{d}{d t} \frac{1}{i} \frac{\delta U_{0}\left[s \mid t_{f}, t_{i}\right]}{\delta J_{r}(t)}=(2 \pi)^{-N} \lim _{t^{\prime}+t_{-}} \int_{-\infty}^{+\infty} d x d y\left[a_{r_{\alpha}}(x, y) \Delta_{F}(x, y ;-\alpha)\right] U_{0}\left[s \mid t_{f}, t_{i}\right]-K_{r}(t) U_{0}\left[s \mid t_{f}, t_{i}\right], \\
& \frac{d}{d t} \frac{1}{i} \frac{\delta U_{0}\left[s \mid t_{f}, t_{i}\right]}{\delta K_{r}(t)}=(2 \pi)^{-N} \lim _{t^{\prime} \rightarrow t_{-}} \int_{-\infty}^{+\infty} d x d y\left[b_{r_{\alpha}}(x, y) \Delta_{F}(x, y ;-\alpha)\right] U_{0}\left[s \mid t_{f}, t_{i}\right]+J_{r}(t) U_{0}\left[s \mid t_{f}, t_{i}\right],
\end{aligned}
$$

where

$$
\Delta_{F}(x, y ;-\alpha)=(2 \pi)^{-N} \int_{-\infty}^{+\infty} d u d v \exp \left[i\left(\frac{1}{2}-\alpha\right) u \cdot v\right] \exp \left\{i\left[x-\frac{1}{i} \frac{\delta}{\delta J(t)}\right] \cdot u\right\} \exp \left\{i\left[y-\frac{1}{i} \frac{\delta}{\delta K\left(t^{\prime}\right)}\right] \cdot v\right\},
$$

and

$$
U_{0}\left[s \mid t_{f}, t_{i}\right]=\left\langle E_{0}\left|U\left[s \mid t_{f}, t_{i}\right]\right| E_{0}\right\rangle .
$$

Obviously, we are assuming that the operator $H$ is bounded from below; in order to define a physically sensible problem. In (2.24) we have denoted by $\left|E_{0}\right\rangle$ the ground state of $H$. In the literature, $U_{0}$ is known as the Green's function generating functional.

Thus, we have demonstrated that the phase-space functional differential formalism can only be settled, in the more general case, in terms of a discrete procedure. Since the replacements (2.12a) and (2.12b) do not introduce an effective $\alpha$ dependence into Schwinger's equations, their correspondent solutions will not depend upon $\alpha$. Nevertheless, one must always choose some $\alpha$ discrete procedure for expressing the problem as a system of functional differential equations. This situation somehow resembles that found in gauge field theories; to solve the problem one must choose a gauge, but the physically meaningful results are gauge independent.

We shall next carry out the integration of Eqs. (2.22). In functional sense, the set of equations (2.22) is linear. Its solution can, then, be written in the form of a functional Fourier transform, i.e.,

$$
\begin{aligned}
U_{0}\left[s \mid t_{f}, t_{i}\right]= & \Re \int_{-\infty}^{+\infty} d q_{f} d q_{i} \Phi_{0}^{*}\left(q_{f}\right) \Phi_{0}\left(q_{i}\right) \\
\times & \int[D q][D p] \tilde{U_{0}}\left[\tilde{s} \mid t_{f}, t_{i}\right] \\
& \times \exp \left\{i \int_{t_{i}}^{t_{f}} d t[J(t) \cdot q(t)+K(t) \cdot p(t)]\right\},
\end{aligned}
$$


where $q(t)$ and $p(t)$, generically denoted by $\tilde{s}$, are the Fourier transformed variables of $J(t)$ and $K(t)$, respectively, $\tilde{U}_{0}$ is the functional Fourier transform of $U_{0}, \mathfrak{N}$ is a normalization constant and $\Phi_{0}(q)=\left\langle q \mid E_{0}\right\rangle$. No statement is made about the definition adopted for the continuous functional integral in (2.25). After replacing (2.25) into (2.22) one arrives at

$i \tilde{U}_{0}\left[\tilde{s} \mid t_{f}, t_{i}\right]\left[\frac{d q_{r}(t)}{d t}-\Omega_{r}^{(a)}(q(t), p(t))\right]=\frac{\delta \tilde{U}_{0}\left[\tilde{s} \mid t_{f}, t_{i}\right]}{\delta p_{r}(t)}$,

$i \tilde{U}_{0}\left[\tilde{s} \mid t_{f}, t_{i}\right]\left[-\frac{d p_{r}(t)}{d t}+\Omega_{r}^{(b)}(q(t), p(t))\right]=\frac{\delta \tilde{U}_{0}\left[\tilde{s} \mid t_{f}, t_{i}\right]}{\delta q_{r}(t)}$,

where

$$
\begin{aligned}
& \Omega_{r}^{(a)}(q(t), p(t)) \\
& \quad=\exp \left[-\frac{i q(t) \cdot p(t)}{\left(\frac{1}{2}-\alpha\right)}\right] a_{r_{\alpha}}(\phi, \xi) \exp \left[\frac{i q(t) \cdot p(t)}{\left(\frac{1}{2}-\alpha\right)}\right],
\end{aligned}
$$

$\Omega_{r}^{(b)}(q(t), p(t))$

$$
=\exp \left[-\frac{i q(t) \cdot p(t)}{\left(\frac{1}{2}-\alpha\right)}\right] b_{r_{\alpha}}(\phi, \xi) \exp \left[\frac{i q(t) \cdot p(t)}{\left(\frac{1}{2}-\alpha\right)}\right],
$$

$\phi_{k}=-i\left(\frac{1}{2}-\alpha\right) \frac{\partial}{\partial p_{k}(t)}$,

$\xi_{k}=-i\left(\frac{1}{2}-\alpha\right) \frac{\partial}{\partial q_{k}(t)}$.

One can easily convince oneself that $\Omega_{r}^{(a)}$ and $\Omega_{b}^{(b)}$ are, in fact, $\alpha$ independent. To illustrate this point we shall work out two explicit examples. First, we take

$$
H(Q, P)=\frac{1}{2} P^{2}+\omega^{2} Q^{2}+\frac{1}{2} \omega(Q P+P Q),
$$

whose corresponding $\mathrm{GWT}_{\alpha}$ is found to be [see Eq. (2.14)]

$$
h_{\alpha}(q, p)=\frac{1}{2} p^{2}+\omega^{2} q^{2}+\omega q p-i \alpha \omega .
$$

Then,

$$
\begin{aligned}
& a_{\alpha}(\phi, \xi)=\omega \phi+\xi, \\
& b_{\alpha}(\phi, \xi)=-2 \omega^{2} \phi-\omega \xi .
\end{aligned}
$$

After using (2.28) one finds that

$$
\begin{aligned}
& \Omega^{(a)}(q(t), p(t))=\omega q(t)+p(t), \\
& \Omega^{(b)}(q(t), p(t))=-2 \omega^{2} q(t)-\omega p(t),
\end{aligned}
$$

Therefore, all coefficient functions in (2.26) are $\alpha$ independent and, as a consequence, so is $\tilde{U}_{0}$.

Our second, and last, example is slightly more complicated. This time we take

$$
H(Q, P)=Q P^{2} Q,
$$

whose GWT $\mathrm{GW}_{\alpha}$ is found to be

$$
h_{\alpha}(q, p)=q^{2} p^{2}-4 i \alpha q p+\left(\frac{1}{2}-2 \alpha^{2}\right) \text {. }
$$

Then,

$$
\begin{aligned}
& a_{\alpha}(\phi, \xi)=2 \phi^{2} \xi-4 i \alpha \phi, \\
& b_{\alpha}(\phi, \xi)=-2 \phi \xi^{2}-4 i \phi \xi .
\end{aligned}
$$

After carrying out the replacements indicated in (2.28) one arrives at

$$
\begin{aligned}
& \Omega^{(a)}(q(t), p(t))=2 q^{2}(t) p(t)-2 i q(t), \\
& \Omega^{(b)}(q(t), p(t))=-2 q(t) p^{2}(t)+2 i p(t) .
\end{aligned}
$$

Once again, we verify that Eqs. (2.26) do not contain $\alpha$ dependent coefficient functions.

The $\alpha$ independence of $\tilde{U}_{0}[\tilde{s}]$ guarantees, through (2.25), that the generating functional $U_{0}[s]$ does not depend upon the discrete procedure as it must.

We close this section by noticing that any explicit solution of Schwinger's equations, in spite of being $\alpha$ independent, will always involve a specific discrete process. For example, Eqs. (2.26) acquire a rather simple form for $\alpha=+\frac{1}{2}$ (standard) ordering) whose integration yields

$$
\begin{aligned}
\tilde{U}_{0}\left[s \mid t_{f}, t_{i}\right]=\exp \left\{i \int_{t_{i}}^{t_{f}} d t[\dot{q}(t) \cdot p(t)\right. & \\
& \left.\left.-h_{1 / 2}(q(t), p(t))\right]\right\} .
\end{aligned}
$$

Here, $\tilde{U}_{0}$ is given in terms of $h_{1 / 2}$ which is a specific GWT of $H$. Any other $\alpha$ can be brought into (2.37) by using

$$
h_{\alpha}(q, p)=\exp \left(-i \alpha \frac{\partial}{\partial q} \cdot \frac{\partial}{\partial p}\right) h_{0}(q, p)
$$

This last expression follows directly from Eqs. $(2.14)-(2.16)$ and it is a generalization of a similar relation found by Mizrahi ${ }^{20}$ for Weyl's ${ }^{21}$ ordering $(\alpha=0)$.

Thus, we conclude that the existence of a multiplicity of representations for the quantum-mechanical propagator is not a peculiarity of the timeslicing definition of the path integral but rather a necessity of the functional approach.

What does depend upon the path-integral definition is the operational level at which the $\alpha$ dependence is canceled out. Indeed, for the continuous functional formulation presented in this paper the integrand in (2.30), i.e., $\tilde{U}_{0}$, is already independent of $\alpha$. On the other hand, when the time-slic- 
ing method is used the cancellation of $\alpha$-dependent terms only occurs after performing the functional integrations. This behavior originates in the unavoidable sequence of approximations one must undergo for deriving the path-integral representation of the propagator via the time-slicing procedure. These approximations become exact in the continuous limit and it is there that the $\alpha$ dependence disappears. ${ }^{10,14,15}$

\section{FINAL REMARKS}

In this paper we have been concerned with the functional formulation of quantum dynamics for a system involving only true canonical variables. The problem posed by constrained variables is out of the scope of this work. Schwinger's equations for constrained systems have been formally derived by Fradkin et al. ${ }^{22}$ but the rules of correspondence for this case were not found by these authors. As a consequence, the severe problem of ordering, characteristic of constrained variables ${ }^{23}$ was left out of consideration in Ref. 22. We hope to discuss these questions in a future publication.

\section{ACKNOWLEDGMENT}

This paper was supported in part by the Conselho Nacional de Desenvolvimento Científico e Tecnológico (Brasil).
${ }^{1}$ L. Cohen, J. Math. Phys. 7, 781 (1966).

${ }^{2}$ G. S. Agarwal and E. Wolf, Phys. Rev. D 2, 2161 (1970); 2, 2187 (1970); 2, 2206 (1970).

${ }^{3}$ I. W. Mayes and J. S. Dowker, Proc. R. Soc. London A327, 131 (1972).

${ }^{4}$ L. Cohen, J. Math. Phys. 17, 597 (1976).

${ }^{5}$ R. Fanelli, J. Math. Phys. 17, 490 (1976).

${ }^{6}$ J. S. Dowker, J. Math. Phys. 17, 1873 (1976).

${ }^{7}$ J.-L. Gervais and A. Jevicki, Nucl. Phys. B110, 93 (1976).

${ }^{8}$ H. O. Girotti and Th. A. J. Maris, Report No. IFUFRGS, 1977 (unpublished).

${ }^{9}$ H. Leschke and M. Schmutz, Z. Phys. B27, 85 (1977).

${ }^{10}$ A. C. Hirshfeld, Phys. Lett. 67A, $5(\overline{1978)}$;

H. Leschke, A. C. Hirshfeld, and T. Suzuki, Phys. Rev. D 18, 2834 (1978); H. Leschke, A. C. Hirshfeld, and T. Suzuki, Phys. Lett. 67A, 87 (1978).

${ }^{11} \mathrm{~J}$. Bertrand and M. Irac, Lett. Math. Phys. $\underline{3}, 97$ (1979).

${ }^{12}$ R. P. Feynman, Rev. Mod. Phys. 20, 367 (1948); R. P. Feynman and A. R. Hibbs, in Quantum Mechanics and Path-Integrals (McGraw-Hill, New York, 1965).
${ }^{13}$ C. Garrod, Rev. Mod. Phys. 38, 483 (1966).

${ }^{14} \mathrm{~F}$. Langouche, D. Roekaerts, and E. Tirapegui, Physica (Utrecht) 95A, 252 (1979); Nuovo Cimento 53B, 135 (1979); Prog. Theor. Phys. 6, 1617 (1979).

${ }^{15}$ F. Langouche, D. Roekaerts, and E. Tir apegui, Phys. Rev. D 20, 419 (1979).

${ }^{16} \mathrm{C}$. DeWitt-Morette, A. Maheshwari, and B. Nelson, Phys.Rep. 50C, 255 (1979).

${ }^{17}$ M. Mizrahi, J. Math. Phys. 17, 566 (1976); 19,298 (1978).

${ }^{18} \mathrm{~J}$. Schwinger, Proc. Nat. Acad. Sci. 37, 452 (1951); 37,455 (1951).

${ }^{19} \mathrm{~A}$. Visconti, in Théorie Quantique des Champs (Gauthiers-Villars, Paris, 1965).

${ }^{20}$ M. Mizrahi, J. Math. Phys. 16, 2201 (1975).

${ }^{21} \mathrm{H}$. Weyl, Z. Physik 46, 1 (1927); in Theory of Groups and Quantum Mechanics (Dover, New York, 1950).

${ }^{22}$ E. S. Fradkin and G. A. Vilkovisky, Report No. CERN TH 2332, 1977 (unpublished).

${ }^{23}$ P. A. M. Dirac, in Lectures in Quantum Mechanics (Belfer Graduate School of Science, Yeshiva University, New York, 1964). 